\title{
LONG MONOTONE PATHS ON SIMPLE 4-POLYTOPES
}

\author{
JULIAN PFEIFLE
}

\begin{abstract}
The Monotone Upper Bound Problem (Klee, 1965) asks if the number $M(d, n)$ of vertices in a monotone path along edges of a $d$-dimensional polytope with $n$ facets can be as large as conceivably possible: Is $M(d, n)=M_{\mathrm{ubt}}(d, n)$, the maximal number of vertices that a $d$-polytope with $n$ facets can have according to the Upper Bound Theorem?

We show that in dimension $d=4$, the answer is "yes", despite the fact that it is "no" if we restrict ourselves to the dual-to-cyclic polytopes. For each $n \geq 5$, we exhibit a realization of a polar-to-neighborly 4-dimensional polytope with $n$ facets and a Hamilton path through its vertices that is monotone with respect to a linear objective function.

This constrasts an earlier result, by which no polar-to-neighborly 6-dimensional polytope with 9 facets admits a monotone Hamilton path.
\end{abstract}

\section{INTRODUCTION}

While investigating the complexity of the simplex algorithm for linear programming, Klee [4] in 1965 posed the Monotone Upper Bound Problem: For $n>d \geq 2$, he asked for the maximal number $M(d, n)$ of vertices of a $d$-dimensional polytope with $n$ facets that can lie on a monotone path, i.e., on a path along edges that is strictly increasing with respect to a linear objective function.

McMullen's 1971 Upper Bound Theorem [5] (claimed by Motzkin [6] in 1957) states that the maximal number $M_{\text {ubt }}(d, n)$ of vertices that any $d$-dimensional polytope with $n$ facets can have is achieved by the polars $C_{d}(n)^{\Delta}$ of cyclic $d$-polytopes with $n$ facets.

The Upper Bound Theorem yields, for all $n>d \geq 2$, the inequality

$$
M(d, n) \leq M_{\mathrm{ubt}}(d, n),
$$

but from this it is not clear whether equality always holds, that is, if for all $n>d \geq 2$ one can construct a simple polar-to-neighborly $d$-polytope with $n$ facets that admits a monotone Hamilton path with respect to a linear objective function. Equality in (1) is known in the cases $d \leq 3$ and $n \leq d+2$.

Date: February 16, 2004.

2000 Mathematics Subject Classification. 52B12; 52B05.

The author was financed by the DFG Graduiertenkolleg Combinatorics, Geometry, and Computation (GRK 588-2), the GIF project Combinatorics of Polytopes in Euclidean Spaces (I-624-35.6/1999), and post-doctoral fellowships from MSRI and Institut de Matemàtica de la Universitat de Barcelona. 
However, in [7] we show that in fact $M(6,9)<M_{\mathrm{ubt}}(6,9)$ : there exists no realization of the (combinatorially unique) polar-to-neighborly 6-polytope $C_{6}(9)^{\Delta}$ with 9 facets and 30 vertices that admits such a monotone Hamilton path.

For the parameters $d=4, n=8$, one can show using the same (basically combinatorial) methods that there is also no realization of $C_{4}(8)^{\Delta}$ with a monotone Hamilton path — but as we will show here, there are other dual-to-neighborly but not dual-to-cyclic 4-dimensional polytopes with 8 facets that admit a realization with a monotone path through all vertices.

In fact, in this paper we prove considerably more: we provide a geometric construction that shows that the inequality (1) is tight in dimension $d=4$ for all $n \geq 5$.

Main Theorem. For each integer $m \geq 0$, there exists a simple polar-to-neighborly 4dimensional polytope $Q_{m}$ with $n=m+5$ facets and a linear objective function $f: \mathbb{R}^{4} \rightarrow \mathbb{R}$, such that the orientation induced by $f$ on the 1-skeleton of $Q_{m}$ admits a monotone Hamilton path. Therefore,

$$
M(4, n)=M_{\mathrm{ubt}}(4, n)=\frac{1}{2} n(n-3) .
$$

In other words, the maximal number $M(4, n)$ of vertices on a strictly monotone path in the graph of a 4-dimensional polytope with $n$ facets equals the maximal number of vertices that such a polytope can have according to the Upper Bound Theorem.

An interesting feature used in our proof is that for $m \geq 3$, the (polar-to-)neighborly polytopes $Q_{m}$ are not polar to cyclic ones. In fact, exhaustive enumeration shows that already the graph of $C_{4}(8)^{\Delta}$ does not satisfy a combinatorial condition necessary for the existence of an monotone path, namely, it does not admit a Hamilton AOF Holt-Klee orientation [2]. This is also true for the graphs of the polytopes $C_{4}(n)^{\Delta}$ for $8 \leq n \leq 12$; we conjecture that the graphs of $C_{4}(n)^{\Delta}$ for all $n \geq 8$ admit no Hamilton AOF Holt-Klee orientation.

The structure of the paper is as follows: We first give an explicit description, reminiscent of Gale's Evenness Criterion for polar-to-cyclic polytopes, of the combinatorial structure of a family $\left\{Q_{m}^{d}: d \geq 4\right.$ even, $\left.m \geq 0\right\}$ of simple (polar-to-)neighborly $d$-dimensional polytopes with $m+d+1$ facets (Sections 2 and 3). For $d=4$, we then use this description to specify a Hamilton path $\pi_{m}$ on each $Q_{m}:=Q_{m}^{4}$ (Section 4). In Section 5, we start with a monotone path $\pi_{0}$ on a certain realization of the 4-simplex $Q_{0}$, and for $m \geq 0$ inductively realize the polytope $Q_{m+1}$ in such a way that the path $\pi_{m+1}$ is strictly monotone with respect to a suitable objective function (Theorem 2.5). We proceed in three steps: First, we position $Q_{m}$ in a suitable way with respect to the standard coordinates on $\mathbb{R}^{4}$ (Section 5.4). We then find a "cutting plane" $H_{m+1}$ such that the polytope $Q_{m} \cap H_{m+1}^{\geq 0}$ has the right combinatorial type (Section 5.5). Finally, we complete the construction in Section 5.6 by applying a projective transformation $\psi$ to $\mathbb{R}^{4}$ such that the path $\psi\left(\pi_{m}\right)$ on $Q_{m+1}:=\psi\left(Q_{m} \cap H_{m+1}^{\geq 0}\right)$ is strictly monotone with respect to the objective function $f: \mathbb{R}^{4} \rightarrow \mathbb{R}, \boldsymbol{x} \mapsto x_{4}$. 


\section{MAin RESUlts}

Theorem 2.1 (modified Gale's Evenness Criterion). For each $m \geq 0$ and even $d \geq 4$, the following sets correspond to the vertices of a combinatorial type $\widetilde{Q}_{m}^{d}$ of a simple $d$ dimensional polar-to-neighborly polytope with $n=m+d+1$ facets.

$\triangleright$ Type 1. The union of one "triplet with a hole" and $d / 2-1$ pairs of indices

$$
\left\{j_{1}, j_{1}+2\right\} \cup\left\{j_{2}, j_{2}+1\right\} \cup \cdots \cup\left\{j_{d / 2}, j_{d / 2}+1\right\},
$$

where $1 \leq j_{1}<n-d+1, j_{1}+3 \leq j_{2}, j_{k}+2 \leq j_{k+1}$ for $2 \leq k \leq d / 2-1$, and $j_{d / 2}<n$.

$\triangleright$ Type $2 \mathrm{a}$. The union of one triplet, the singleton $\{n\}$, and $d / 2-2$ pairs of indices

$$
\left\{j_{1}, j_{1}+1, j_{1}+2\right\} \cup\left\{j_{2}, j_{2}+1\right\} \cup \cdots \cup\left\{j_{d / 2-1}, j_{d / 2-1}+1\right\} \cup\{n\},
$$

where $1 \leq j_{1}<n-d+1, j_{1}+3 \leq j_{2}, j_{k}+2 \leq j_{k+1}$ for $2 \leq k \leq d / 2-2$, and $j_{d / 2-1}<n-1$.

$\triangleright$ Type $2 \mathrm{~b}$. The union of $d / 2$ pairs of indices

$$
\{1,2\} \cup\left\{j_{1}, j_{1}+1\right\} \cup \cdots \cup\left\{j_{d / 2-1}, j_{d / 2-1}+1\right\},
$$

where $3 \leq j_{1}, j_{k}+2 \leq j_{k+1}$ for $2 \leq k \leq d / 2-2$, and $j_{d / 2-1}<n$.

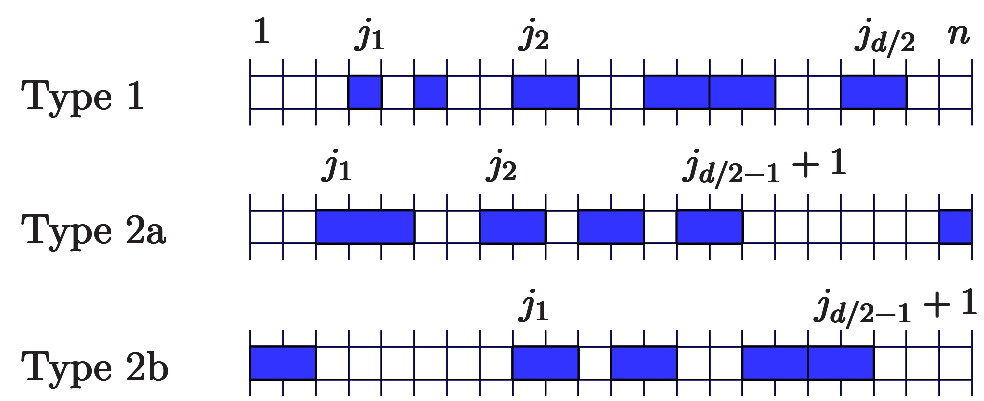

Figure 1: The vertex-facet incidences of the polytopes $\widetilde{Q}_{m}^{d}$ are obtained from these patterns by fixing the dark boxes, and sliding the lighter boxes between 1 and $n$ without overlap. For Type 1 , the box $\{i, i+2\}$ must be regarded as one rigid unit.

Remark 2.2. If we accept for the moment the existence of the polytopes $\widetilde{Q}_{m}^{d}$, it is easy to verify that they are polar-to-neighborly by counting the number of vertices using Figure 1:

$$
\begin{aligned}
f_{0}\left(\widetilde{Q}_{n-d-1}^{d}\right) & =\underbrace{\left(\begin{array}{c}
n-2-(d / 2-1) \\
d / 2
\end{array}\right)}_{\text {Type } 1}+\underbrace{\left(\begin{array}{c}
n-2-(d / 2-2)-1 \\
d / 2-1
\end{array}\right)}_{\text {Type 2a }}+\underbrace{\left(\begin{array}{c}
n-2-(d / 2-1) \\
d / 2-1
\end{array}\right)}_{\text {Type 2b }} \\
& =\left(\begin{array}{c}
n-1-d / 2 \\
d / 2
\end{array}\right)+2\left(\begin{array}{c}
n-1-d / 2 \\
d / 2-1
\end{array}\right) \\
& =\left(\begin{array}{c}
n-d / 2 \\
d / 2
\end{array}\right)+\left(\begin{array}{c}
n-1-d / 2 \\
d / 2-1
\end{array}\right),
\end{aligned}
$$


which is the number of vertices of a simple polar-to-neighborly $d$-polytope with $n=m+d+1$ facets, since $d$ is assumed even. By [9, Chapter 8], any polytope with that many vertices is polar-to-neighborly.

From now on, we will always write $\widetilde{Q}_{m}:=\widetilde{Q}_{m}^{4}$.

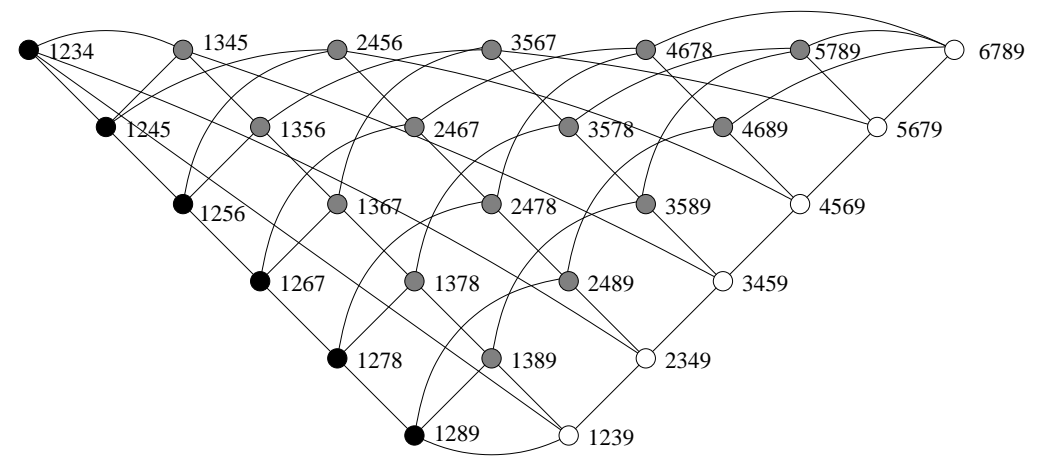

Figure 2: Graph of the 4-polytope $\widetilde{Q}_{4}$ with $n=9$ facets. Vertices of type $1,2 \mathrm{a}$, and $2 \mathrm{~b}$ are drawn in gray, white, and black, respectively. Each vertex is labelled with the facets it is incident to.

Proposition 2.3. Each polytope $\widetilde{Q}_{m}$ admits a Hamilton path $\widetilde{\pi}_{m}$ in its graph that induces an AOF-orientation (cf. Figure 3 and Definition 4.1 below).
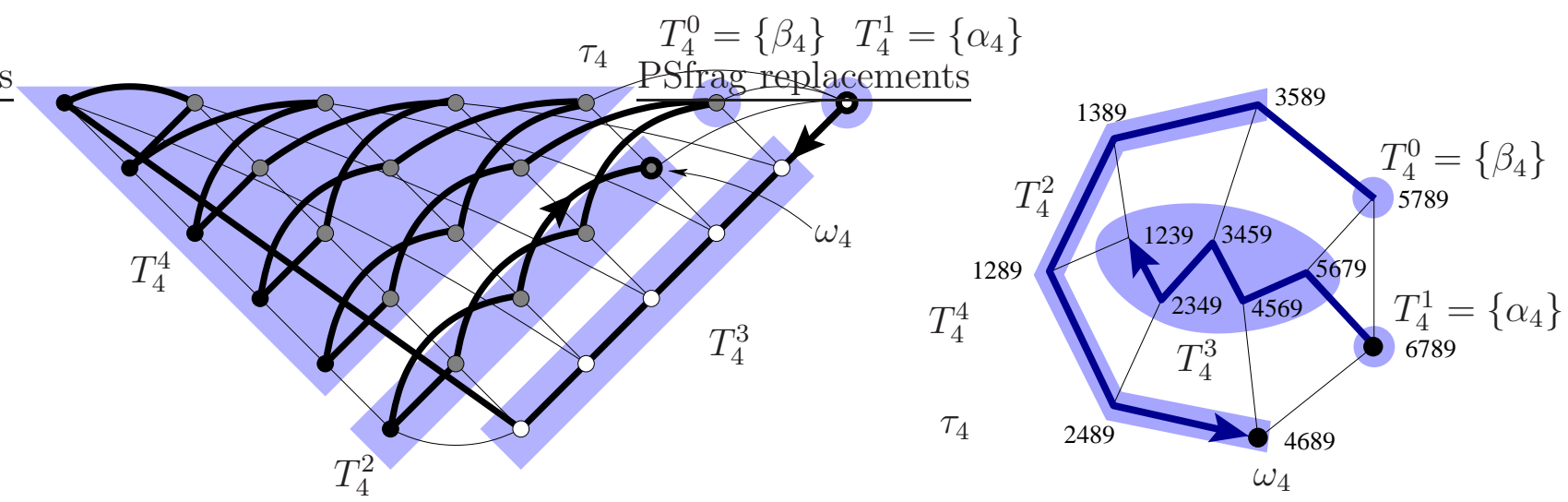

Figure 3: Left: Graph of $\widetilde{Q}_{4}^{4}$. The partition of the vertices into the tips $T^{0}, T^{1}, \ldots, T^{4}$ is shown, along with the Hamilton path $\tilde{\pi}_{4}$ (bold). The source $\alpha_{m}$ is labeled $\{n-3, n-2, n-1, n\}$, and the $\operatorname{sink} \omega_{m}=\{n-5, n-3, n-1, n\}$. See Convention 5.1 for the labels of the other marked vertices. Right: The facet $F_{4}^{3}$ with the restriction of $\tilde{\pi}_{4}$ to it.

Remark 2.4. The crucial property for our realization construction is that the path $\tilde{\pi}_{m}$ begins in a certain facet $F_{m}^{3}$ of the polytope $Q_{m}$ (defined below), traverses the rest of $Q_{m}$, 
and then returns to $F_{m}^{3}$ (cf. Figure 3 ). This permits us to add new vertices to the beginning and end of $\tilde{\pi}_{m}$ by modifying only the facet $F_{m}^{3}$.

Theorem 2.5. There exists a family $\left\{Q_{m}: m \geq 0\right\}$ of special realizations of the combinatorial types $\widetilde{Q}_{m}$, in which each Hamilton path $\pi_{m}$ visits the vertices of $Q_{m}$ in the order given by increasing $x_{4}$-coordinate. This family may be realized inductively starting from the 4-simplex $Q_{0}$ in such a way that for all $m \geq 0$, a realization of $Q_{m+1}$ with a monotone Hamilton path $\pi_{m+1}$ may be obtained from any realization of $Q_{m}$ with such a path $\pi_{m}$.

\section{Constructing the combinatorial types $\widetilde{Q}_{m}^{d}$}

3.1. Facet splitting. We will prove Theorem 2.1 using Barnette's technique of facet splitting [1]. Put briefly, for each even $d \geq 4$ we will inductively construct a family $\left\{\left(\widetilde{Q}_{m}^{d}, \mathcal{F}_{m}\right): m \geq 0\right\}$, where each $\widetilde{Q}_{m}^{d}$ is the combinatorial type of a simple $d$-dimensional polytope with $m+d+1$ facets, and $\mathcal{F}_{m}$ is a flag of faces on $\widetilde{Q}_{m}^{d}$ (to be defined shortly). We then use $\mathcal{F}_{m}$ to find a "good" oriented hyperplane $H_{m+1}$ in general position with respect to the vertices of $\widetilde{Q}_{m}^{d}$, and set $\widetilde{Q}_{m+1}^{d}:=\widetilde{Q}_{m}^{d} \cap H_{m+1}^{\geq 0}$.

Definition 3.1. Let $P$ be a $d$-dimensional simple polytope. A flag of faces on $P$ is a chain

$$
\mathcal{F}: \emptyset=F^{-1} \subset F^{0} \subset F^{1} \subset \cdots \subset F^{d}=P
$$

of faces of $P$ such that $\operatorname{dim} F^{i}=i$ for $i=0,1, \ldots, d$. The $i$-th tip of a flag $\mathcal{F}$ is $T^{i}:=$ vert $F^{i} \backslash \operatorname{vert} F^{i-1}$, for $0 \leq i \leq d$. We say that the tip $T^{i}$ is even resp. odd according to the parity of $i$. Moreover, for $0 \leq k \leq d$ we set

$$
T_{\text {even }}^{\leq k}=\bigcup_{\substack{0 \leq e \leq k \\ e \text { even }}} T^{e} \quad \text { and } \quad T_{\text {odd }}^{\leq k}=\bigcup_{\substack{1 \leq o \leq k \\ o \text { odd }}} T^{o} .
$$

Lemma 3.2. Let $P$ be a simple $d$-dimensional polytope with $n$ facets, and $\mathcal{F}$ a flag of faces as in (2). Then there exists an affine oriented hyperplane $H$ in general position with respect to $P$ such that $T_{\text {even }}^{\leq d} \subset H^{+}$and $T_{\text {odd }}^{\leq d} \subset H^{-}$. In particular, $P \cap H^{\geq 0}$ is a simple $d$-polytope with $n+1$ facets.

Proof. Pick an oriented point $\{v\}=H^{0} \subset \operatorname{relint} F^{1}$ such that $T^{0} \in\left(H^{0}\right)^{+}$. Inductively, for $1 \leq k \leq d-1$, if we have already chosen an oriented $(k-1)$-dimensional affine subspace $H^{k-1}$ in aff $F^{k}$ such that

$$
T_{\text {even }}^{\leq k} \subset\left(H^{k-1}\right)^{+} \quad \text { and } \quad T_{\text {odd }}^{\leq k} \subset\left(H^{k-1}\right)^{-},
$$

we take a $k$-plane $H^{k}$ that initially coincides with aff $F^{k}$, and orient it in such a way that $T^{k+1}$ lies in $\left(H^{k}\right)^{+}$if $k+1$ is even, respectively in $\left(H^{k}\right)^{-}$if $k+1$ is odd. Now we rotate $H^{k}$ by a sufficiently small amount around $H^{k-1}$ in such a way that $T_{\text {even }}^{\leq k} \subset\left(H^{k}\right)^{+}$. Then (3) even holds with $k$ replaced by $k+1$. By construction, the hyperplane $H:=H^{d-1}$ is in general position with respect to $P$. 
Definition 3.3. The family $\left\{\left(\widetilde{Q}_{m}^{d}, \mathcal{F}_{m}\right): m \geq 0\right\}$ of $d$-dimensional polytopes $\widetilde{Q}_{m}^{d}$ equipped with flags $\mathcal{F}_{m}$ of faces is defined in the following way:

(a) $\widetilde{Q}_{0}^{d}$ is the combinatorial type of the $d$-simplex $\operatorname{conv}\left\{v_{1}, v_{2}, \ldots, v_{d+1}\right\}$. The flag $\mathcal{F}_{0}$ is defined by setting $F_{0}^{i}:=\operatorname{conv}\left\{v_{1}, v_{2}, \ldots, v_{i+1}\right\}$ for $i=0,1, \ldots, d$. Then

$$
T_{0}^{i}:=\operatorname{vert}\left(F_{0}^{i}\right) \backslash \operatorname{vert}\left(F_{0}^{i-1}\right)=\left\{v_{i+1}\right\} \quad \text { for } i=0,1, \ldots, d .
$$

(b) For $m \geq 0$, let $H=H_{m+1}$ be the oriented hyperplane given by applying Lemma 3.2 to $P=\widetilde{Q}_{m}^{d}$ and $\mathcal{F}=\mathcal{F}_{m}$, and set $\widetilde{Q}_{m+1}^{d}:=\widetilde{Q}_{m}^{d} \cap H_{m+1}^{\geq 0}$ and (cf. Figure 4)

$$
\begin{aligned}
T_{m+1}^{0} & :=\operatorname{vert}\left(\operatorname{conv}\left(T_{m}^{1} \cup T_{m}^{2}\right) \cap H_{m+1}\right), \\
T_{m+1}^{1} & :=\operatorname{vert}\left(\operatorname{conv}\left(T_{m}^{0} \cup T_{m}^{1}\right) \cap H_{m+1}\right), \\
T_{m+1}^{j} & :=\operatorname{vert}\left(\operatorname{conv}\left(T_{m}^{j+1} \cup \bigcup_{\substack{0 \leq k<j \\
k+j=0 \bmod 2}} T_{m}^{k}\right) \cap H_{m+1}\right) \quad \text { for } j=2,3, \ldots, d-1, \\
T_{m+1}^{d} & :=\bigcup_{0 \leq k \leq d / 2} T_{m}^{2 k} .
\end{aligned}
$$

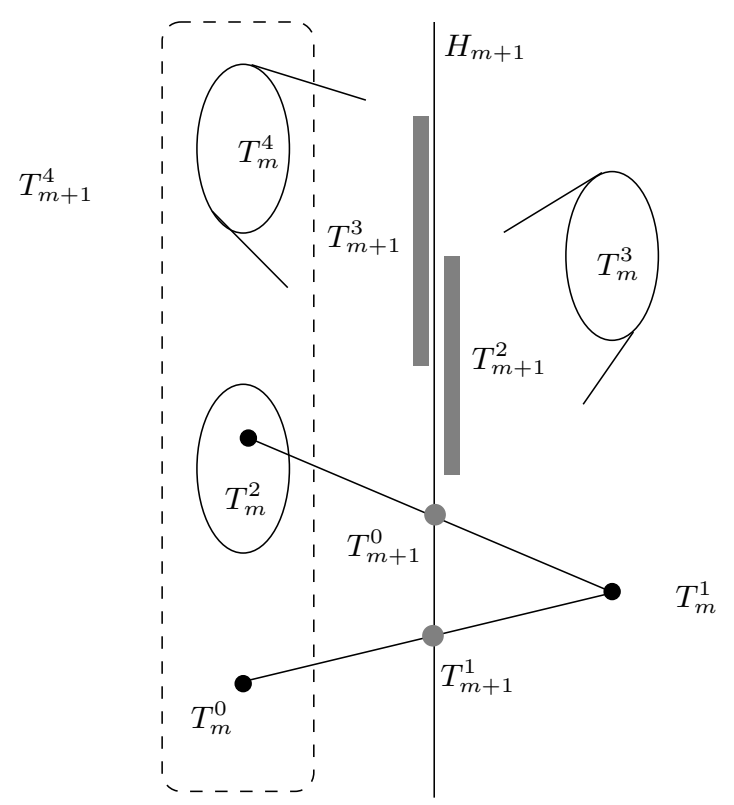

Figure 4: New tips in the case $d=4$.

The flag $\mathcal{F}_{m+1}$ is now defined by $F_{m+1}^{j}:=\bigcup_{i=0}^{j} T_{m+1}^{i}$ for $j=0,1, \ldots, d$. Moreover, put

$$
T_{\text {even }}^{\leq k}(m)=\bigcup_{\substack{0 \leq e \leq k \\ e \text { even }}} T_{m}^{e} \quad \text { and } \quad T_{\text {odd }}^{\leq k}(m)=\bigcup_{\substack{1 \leq o \leq k \\ \text { oodd }}} T_{m}^{o} .
$$


Remark 3.4.

(a) The polytopes $C_{d}(n)^{\Delta}$ arise by exchanging the definitions of $T_{m+1}^{0}$ and $T_{m+1}^{1}$.

(b) All new vertices arise as the intersection of $H_{m+1}$ with some edge $\operatorname{conv}\{v, w\}$ of $\widetilde{Q}_{m}^{d}$, where $v$ and $w$ lie in tips of different parity. Furthermore, all vertices of $\widetilde{Q}_{m}^{d}$ belonging to even tips are also vertices of $\widetilde{Q}_{m+1}^{d}$, and vertices in odd tips disappear.

Proposition 3.5. For each $m \geq 0$, the following is true for the pair $\left(\widetilde{Q}_{m}^{d}, \mathcal{F}_{m}\right)$ :

(a) For all $i, j \in \mathbb{N}$ with $0 \leq i<j \leq d$ and $i+j=1 \bmod 2$ and all $v \in T_{m}^{i}$, there is exactly one $w \in T_{m}^{j}$ such that $\operatorname{conv}\{v, w\} \in \operatorname{sk}^{1}\left(\widetilde{Q}_{m}^{d}\right)$. This gives rise to bijections $T_{\text {even }}^{\leq k}(m) \cong T_{m+1}^{k}$ for odd $0<k<d$ resp. $T_{\text {odd }}^{\leq k}(m) \cong T_{m+1}^{k}$ for even $0 \leq k \leq d$.

(b) $\left|T_{m}^{e}\right|=\left|T_{m}^{e+1}\right|=\left(\begin{array}{c}e / 2+m \\ m\end{array}\right)$ for even $e=0,2, \ldots, d-2$, and $\left|T_{m}^{d}\right|=\left(\begin{array}{c}d / 2+m \\ m\end{array}\right)$. This proves again that $\widetilde{Q}_{m}^{d}$ is polar-to-neighborly.

Proof. (a) This follows because $v$ lies in $F_{m}^{j-1}=\bigcup_{i=0}^{j-1} T_{m}^{i}$, and $\operatorname{conv}\left(F_{m}^{j-1}\right)$ is a $(j-1)$ dimensional face of the simple polytope $\operatorname{conv}\left(F_{m}^{j}\right)=\operatorname{conv}\left(F_{m}^{j-1} \cup T_{m}^{j}\right)$.

(b) We proceed by induction, and can assume that the assertion holds for $m \geq 0$. From the bijections in part (a), we conclude for all even $e=0,2, \ldots, d-2$ that

$$
\left|T_{m+1}^{e+1}\right|=\left|T_{m+1}^{e}\right|=\sum_{\substack{i=0 \\
i \text { even }}}^{e}\left|T_{m}^{i}\right|=\sum_{k=0}^{e / 2}\left|T_{m}^{2 k}\right|=\sum_{k=0}^{e / 2}\left(\begin{array}{c}
k+m \\
m
\end{array}\right)=\left(\begin{array}{c}
e / 2+m+1 \\
m+1
\end{array}\right) .
$$

The calculation for $\left|T_{m+1}^{d}\right|$ is similar. The fact that $\widetilde{Q}_{m}^{d}$ is polar-to-neighborly follows by the same argument as in Remark 2.2, since

$$
\begin{aligned}
f_{0}\left(\widetilde{Q}_{m}^{d}\right) & =\sum_{k=0}^{d / 2}\left(\begin{array}{c}
k+m \\
m
\end{array}\right)+\sum_{k=0}^{\lfloor(d-1) / 2\rfloor}\left(\begin{array}{c}
k+m \\
m
\end{array}\right) \\
& =\left(\begin{array}{c}
m+d / 2+1 \\
d / 2
\end{array}\right)+\left(\begin{array}{c}
m+\lfloor(d-1) / 2\rfloor+1 \\
\lfloor(d-1) / 2\rfloor
\end{array}\right) \\
& =\left(\begin{array}{c}
n-d / 2 \\
d / 2
\end{array}\right)+\left(\begin{array}{c}
n-\lceil(d-1) / 2\rceil-1 \\
\lfloor(d-1) / 2\rfloor
\end{array}\right) .
\end{aligned}
$$

\subsection{Combinatorics of the family $\widetilde{Q}_{m}^{d}$.}

Convention 3.6. We introduce labelings to make the combinatorics of the $\widetilde{Q}_{m}$ explicit:

(a) For any labeling of the facets of a simple $d$-polytope $P$ with labels in $[n]:=\{1,2, \ldots, n\}$, let $\lambda$ : vert $P \rightarrow\left(\begin{array}{c}{[n]} \\ d\end{array}\right)$ assign to each vertex $v$ of $P$ the set of labels of all facets that $v$ is incident to. We identify a vertex $v$ with its label $\lambda(v)$.

(b) The facets of the $d$-simplex $\widetilde{Q}_{0}^{d}$ on the vertex set $\left\{v_{1}, v_{2}, \ldots, v_{d+1}\right\}$ are labeled in such a way that $v_{1} \equiv \lambda\left(v_{1}\right)=[d+1] \backslash\{2\}, v_{2}=[d+1] \backslash\{1\}$, and $v_{j}=[d+1] \backslash\{j\}$ for $j=3,4, \ldots, d+1$ (cf. Figure 5 ). 
(c) The "new" facet $\widetilde{Q}_{m}^{d} \cap H_{m+1}$ of $\widetilde{Q}_{m+1}^{d}$ is labelled $m+d+2$.

$$
\begin{aligned}
& \left\{v_{5}\right\}=T_{0}^{4} \quad 2 \mathrm{~b} \quad 12 \mid 34 \\
& \left\{v_{3}\right\}=T_{0}^{2} \quad 2 \mathrm{~b} \quad 12 \mid 45 \\
& \left\{v_{1}\right\}=T_{0}^{0} \quad 1 \quad 13 \mid 45 \\
& 123 \mid 5 \quad 2 \mathrm{a} \quad T_{0}^{3}=\left\{v_{4}\right\} \\
& 234 \mid 5 \quad 2 \mathrm{a} \quad T_{0}^{1}=\left\{v_{2}\right\}
\end{aligned}
$$

Figure 5: The labeling of the vertices of the 4-simplex $\widetilde{Q}_{0}$ according to Convention 3.6(b). Also shown is the classification of the vertices into types 1, 2a, 2b as in Proposition 3.7.

Proposition 3.7. Let $m \geq 0$ and $n=m+d+1$. A vertex $v$ of $\widetilde{Q}_{m}^{d}$ lies in $T_{m}^{i}$ exactly if

$$
\max _{n} \bar{v}:=\max ([n] \backslash v)= \begin{cases}m+2 & \text { for } i=0 \\ m+1 & \text { for } i=1 \\ m+i+1 & \text { for } 2 \leq i \leq d\end{cases}
$$

Proof. This is true for $m=0$ by (4) and Convention 3.6, see also Figures 5 and 6. For $m>0$ and $2 \leq i \leq d-1$, the statement follows because any vertex $\tilde{v} \in T_{m}^{i}$ is of the form $\tilde{v}=\operatorname{conv}\{v, w\} \cap H_{m} \equiv(v \cap w) \cup\{n\}$ for some $v \in T_{m-1}^{k}$ and $w \in T_{m-1}^{i+1}$ with $k \leq i$. But then by induction,

$$
\max _{n-1} \bar{v}<\max _{n-1} \bar{w}=(m-1)+(i+1)+1=m+i+1,
$$

so $\max ([n] \backslash \tilde{v})=m+i+1$ as required. The case $i=d$ follows directly from Definition 3.3, and the cases $i=0,1$ are checked similarly.

Proof of Theorem 2.1. The existence of the family $\left\{\left(\widetilde{Q}_{m}^{d}, \mathcal{F}_{m}\right): m \geq 0\right\}$ follows from Lemma 3.2. Using Propositions 3.5 and 3.7, it is somewhat tedious but elementary to verify that for all $m \geq 0$, the vertices of $\widetilde{Q}_{m}^{d}$ are of the given types. More precisely, all vertices of $T_{\text {even }}^{\leq d}(m)$ are of type 1 or $2 \mathrm{~b}$, and $T_{\text {odd }}^{\leq d-1}(m)$ is made up entirely of vertices of type 2a, cf. Figure 6.

\section{A Hamilton path $\widetilde{\pi}_{m}$ that induces an AOF-ORIENTation on $\widetilde{Q}_{m}$}

Definition 4.1. Let $P$ be a simple $d$-polytope. An acyclic orientation of the graph of $P$ that has a unique sink in each face (including $P$ itself) is called an AOF-orientation on $P$. For any orientation $\mathcal{O}$ of the graph of $P$ and $0 \leq k \leq d$, denote by $h_{k}(\mathcal{O})$ the number of vertices of in-degree $k$ in $\mathcal{O}$.

Proposition 4.2. (see e.g. [9, Chap. 8.3] and [3]) An acyclic orientation $\mathcal{O}$ of the graph of a simple $d$-polytope $P$ is an AOF-orientation if and only if the $h$-vector of $P$ coincides with the vector $\left(h_{0}(\mathcal{O}), h_{1}(\mathcal{O}), \ldots, h_{d}(\mathcal{O})\right)$. 


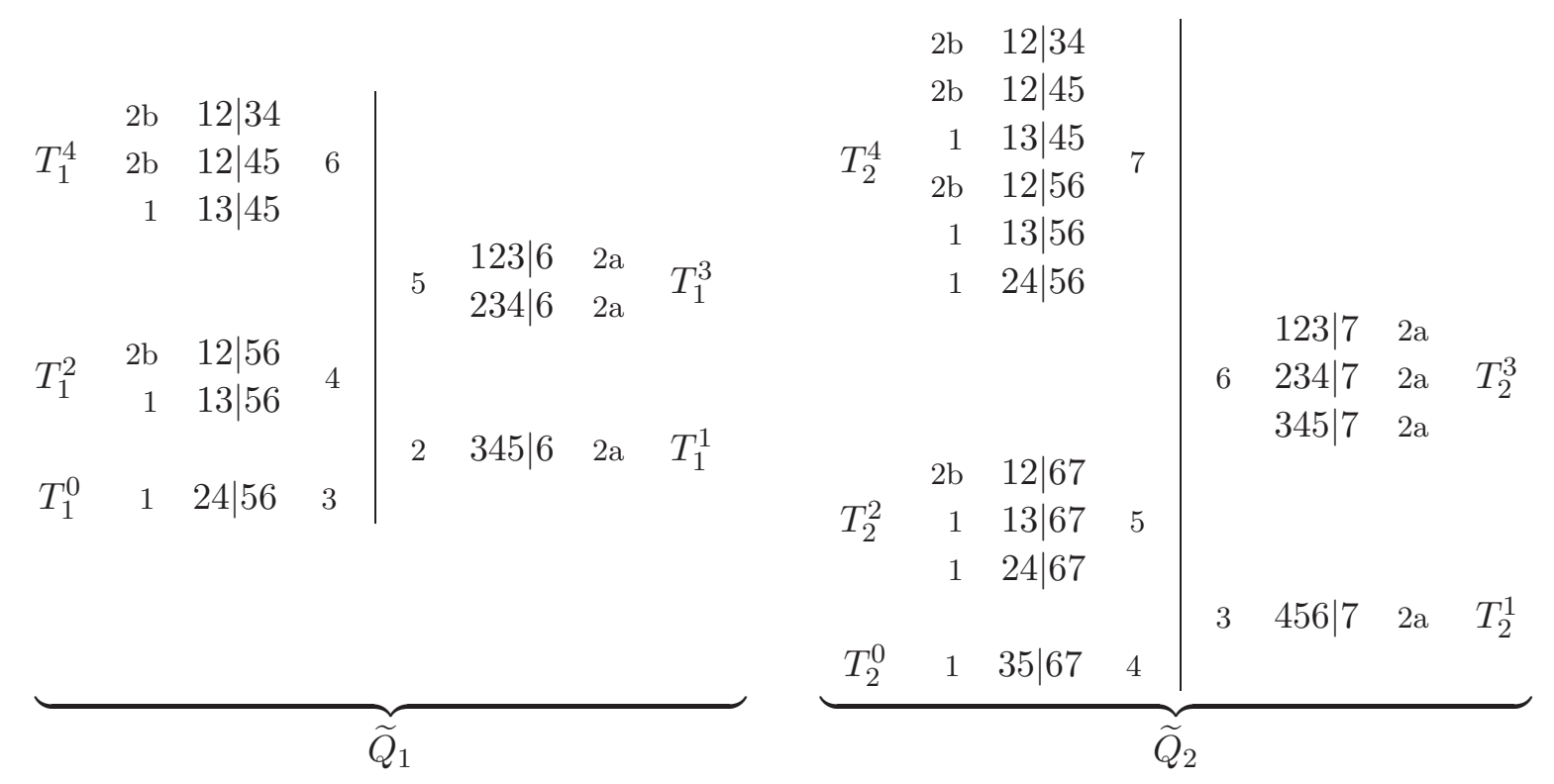

Figure 6: Vertex labels in the polytopes $\widetilde{Q}_{1}$ (left) and $\widetilde{Q}_{2}$ (right). Also shown are the type (outside) of each vertex $v$ and the value of $\max _{n} \bar{v}$ (inside).

Proof of Proposition 2.3. By inspection of Figures 2 and 3, the algorithm of Figure 7 yields a Hamilton path $\widetilde{\pi}_{m}$ in the graph of $\widetilde{Q}_{m}$. Note that $\widetilde{\pi}_{m}$ passes through $T_{m}^{1}, T_{m}^{3}, T_{m}^{4}, T_{m}^{0}$, and $T_{m}^{2}$, in this order (cf. Remark 2.4).

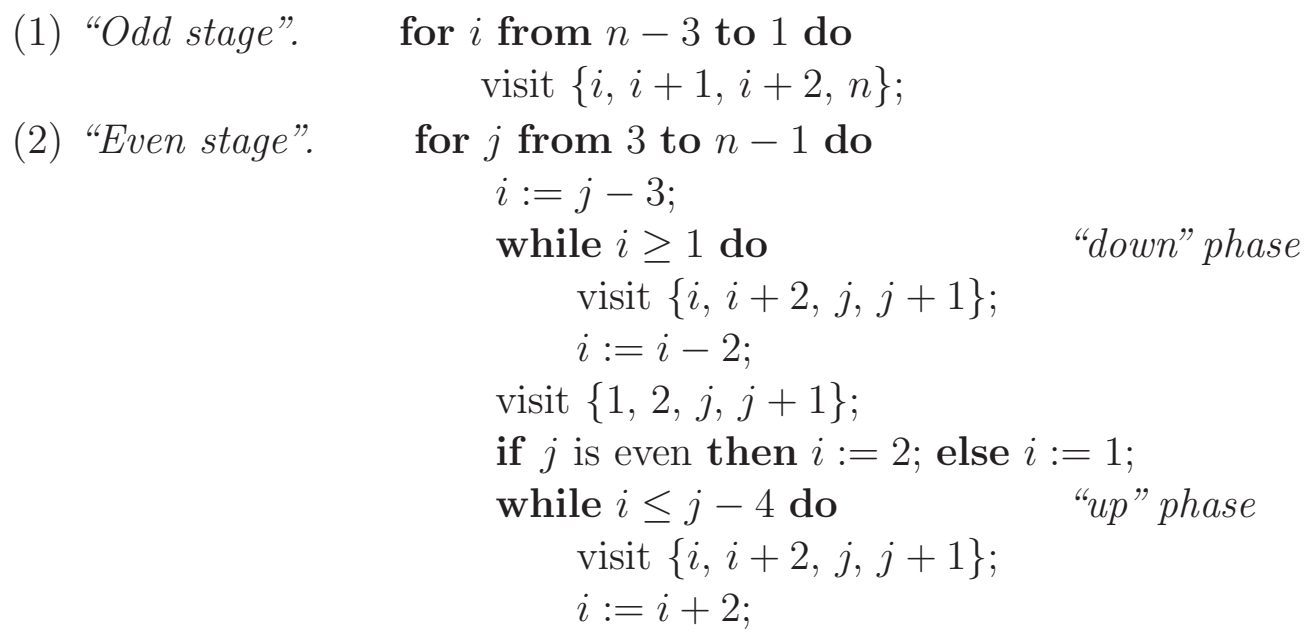

Figure 7: A Hamilton path $\widetilde{\pi}_{m}$ on the graph of $\widetilde{Q}_{m}$ that induces an AOF-orientation $(n:=m+5)$.

We now verify that $\widetilde{\pi}_{m}$ induces an AOF orientation on the graph of $\widetilde{Q}_{m}$. The $h$-vector of a simple polar-to-neighborly $d$-dimensional polytope with $n=m+d+1$ facets is given 
by $h_{k}=\left(\begin{array}{c}n-d-1+k \\ k\end{array}\right)=\left(\begin{array}{c}m+k \\ k\end{array}\right)$ for $k=0,1, \ldots, d$. Therefore, by Proposition 3.5,

$$
\left(\left|T_{m}^{1}\right|,\left|T_{m}^{3}\right|,\left|T_{m}^{4}\right|,\left|T_{m}^{2}\right|,\left|T_{m}^{0}\right|\right)=\left(h_{0}, h_{1}, h_{2}, h_{3}, h_{4}\right) .
$$

By Proposition 4.2, it suffices to verify using Figure 3 that if the orientation of each edge of the graph of $\widetilde{Q}_{m}$ is consistent with the total ordering induced by $\widetilde{\pi}_{m}$, then the vertices of $T^{1}, T^{3}$, resp. $T^{4}$ all have in-degree 0,1 resp. 2 , furthermore $T^{0}$ and all but one of the vertices of $T^{2}$ have in-degree 3 , and this vertex, the sink, has in-degree 4.

\section{Realizing the monotone Hamilton PAThs}

In this section we prove Theorem 2.5, and therefore our Main Theorem.

5.1. Outline of the inductive construction. For all $m \geq 0$, we first find an oriented hyperplane $H_{m+1}$ that separates the odd part $T_{\text {odd }}^{\leq 4}(m)=T_{m}^{1} \cup T_{m}^{3}$ from the even part $T_{\text {even }}^{\leq 4}(m)=T_{m}^{0} \cup T_{m}^{2} \cup T_{m}^{4}$ of $\pi_{m}$. We then create an intermediate pair $\left(Q_{m+1}^{\prime}, \mathcal{F}_{m+1}^{\prime}\right)$ as in Proposition 3.5: $Q_{m+1}^{\prime}:=Q_{m} \cap H_{m+1}^{\geq 0}$ is a simple polar-to-neighborly polytope of the same combinatorial type as $\widetilde{Q}_{m+1}$, and the flag $\mathcal{F}_{m+1}^{\prime}$ of faces is defined as in Definition 3.3(b).

Our combinatorial model $\widetilde{Q}_{m+1}$ provides us with a Hamilton path $\pi_{m+1}$ on $Q_{m+1}^{\prime}$ that is not yet monotone with respect to the objective function $f: \boldsymbol{x} \mapsto x_{4}$. However, we will choose $H_{m+1}$ in such a way that there exists a pencil

$$
\mathcal{H}=\left\{H_{t}: t \in \mathbb{P}^{1}(\mathbb{R}) \cong \mathbb{R} \cup\{\infty\}\right\}
$$

of hyperplanes in $\mathbb{R}^{4}$ with the following properties:

(S1) The common intersection of all hyperplanes in $\mathcal{H}$ is a 2-flat $R=\bigcap_{t \in \mathbb{P}^{1}(\mathbb{R})} H_{t}$ (the axis of $\mathcal{H})$, and vert $Q_{m+1}^{\prime} \cap R=\emptyset$.

(S2) The pencil $\mathcal{H}$ "sorts the vertices of $Q_{m+1}^{\prime}$ correctly": If $p \in H_{r}$ and $q \in H_{s}$ are vertices of $Q_{m+1}^{\prime}$ with $r, s \neq \infty$ and $p$ precedes $q$ in $\pi_{m+1}$, then $r<s$.

We then apply a projective transformation $\psi$ to $\mathbb{R}^{4} \subset \mathbb{P}^{4}(\mathbb{R})$ that maps $H_{\infty}$ to the hyperplane at infinity. Because the common intersection $R$ of all hyperplanes in $\mathcal{H}$ is also mapped to infinity, the image $\psi\left(\mathcal{H}^{b}\right)=\psi\left(\mathcal{H} \backslash H_{\infty}\right)=\left\{\psi\left(H_{t}\right): t \in \mathbb{R}\right\}$ is a family of parallel affine hyperplanes in $\mathbb{R}^{4}$. The new objective function $f$ is then defined by the common normal vector to the hyperplanes in $\psi\left(\mathcal{H}^{b}\right)$, and the Hamilton path $\psi\left(\pi_{m+1}\right)$ on $Q_{m+1}:=\psi\left(Q_{m+1}^{\prime}\right)$ is strictly monotone with respect to $f_{m+1}$ by (S2).

\subsection{Properties of the family of polytopes.}

Notation 5.1. We use the following names for some special vertices of $\widetilde{Q}_{m}$ :

$\triangleright$ The source $\{n-3, n-2, n-1, n\}$ of $\tilde{\pi}_{m}$ is called $\alpha_{m}$ (so that $\left.T_{m}^{1}=\left\{\alpha_{m}\right\}\right)$.

$\triangleright$ The sink is $\omega_{m}:=\{n-5, n-3, n-1, n\} \in T_{m}^{2}$.

$\triangleright \beta_{m}:=\{n-4, n-2, n-1, n\}\left(\right.$ so that $\left.T_{m}^{0}=\left\{\beta_{m}\right\}\right)$.

$\triangleright \tau_{m}:=\{n-5, n-3, n-2, n-1\} \in T_{m}^{4}$. 


\section{Proposition 5.2.}

(a) The induced subgraph of $s k^{1}\left(Q_{m}\right)$ on $T_{m}^{1} \cup T_{m}^{3}$ is a path of length $m+1$ on the $m+2$ vertices $v_{0}^{m}=\alpha_{m}, v_{1}^{m}, \ldots, v_{m+1}^{m}$, and the induced subgraph on $T_{m}^{2}$ is a path $w_{1}^{m}, w_{2}^{m}, \ldots, w_{m+1}^{m}$.

(b) For $0 \leq i \leq m$, the edge $e_{i}=\operatorname{conv}\left\{v_{i}^{m}, v_{i+1}^{m}\right\}$ in $T_{m}^{3}$ is incident to a 2-face $G_{i}$ of $Q_{m}$ such that the vertices of $G_{i} \backslash e_{i}$ are consecutive in $\pi_{m} \cap T_{m}^{4}$.

(c) For $1 \leq i \leq m$, the edge $f_{i}$ of $Q_{m}$ that connects $w_{i}^{m}$ and $w_{i+1}^{m}$ in $T_{m}^{2} \cap \pi_{m}$ is incident to a quadrilateral $R_{i}$ whose other two vertices are consecutive in $T_{m}^{4} \cap \pi_{m}$.

(d) Set $G(m)=\operatorname{vert} \bigcup_{i=0}^{m} G_{i} \backslash e_{i}$ and $R(m)=\operatorname{vert} \bigcup_{i=1}^{m} R_{i} \backslash f_{i}$. Then $G(m) \cup R(m)=T_{m}^{4}$, and $G(m) \cap R(m)=\tau_{m}$.

Proof. (a) All vertices of $T_{m}^{3}$ are of the form $\{i, i+1, i+2, n\}$ for $1 \leq i \leq n-3$, and the only way for two such vertices $v_{i}^{m}$ and $v_{j}^{m}$ to be adjacent for $i<j$ is to have $j=i+1$. The statement about the $w_{i}^{m}$ follows in a similar way. (b) For $1 \leq i \leq m+1$, the 2-face incident to $v_{m+2-i}=\{i, i+1, i+2, n\}$ and $v_{m+1-i}=\{i+1, i+2, i+3, n\}$ that is the intersection of the facets $i+1$ and $i+2$ consists of the vertices of Figure 8. The claim (b) follows because these vertices form a contiguous segment of $\pi_{m}$, and (c) and (d) from Figure 9 (left).

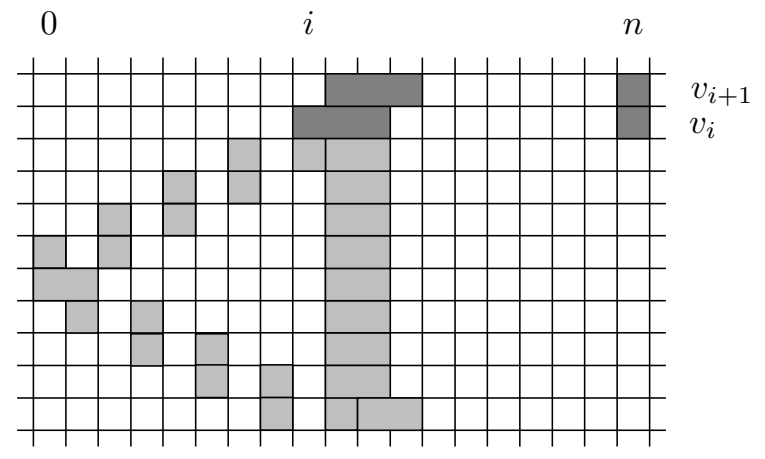

Figure 8: Vertices of a 2-face incident to $v_{i}=\{i, i+1, i+2, n\}$ and $v_{i+1}=\{i+1, i+2, i+3, n\}$ (dark) in $T_{m}^{1} \cup T_{m}^{3}$. The light vertices lie in $T_{m}^{4}$ and form a subpath of $\pi_{m}$.

Observation 5.3. The new start vertex $\alpha_{m+1}$ of $\widetilde{\pi}_{m+1}$ lies on $\operatorname{conv}\left\{\alpha_{m}, \beta_{m}\right\}$, the new end vertex $\omega_{m+1}$ on $\operatorname{conv}\left\{v_{1}^{m}, \beta_{m}\right\}$, and $\beta_{m+1}$ on $\operatorname{conv}\left\{\alpha_{m}, \omega_{m}\right\}$; see Figure 9 (right).

5.3. Start of the induction and inductive invariant. We work in $\mathbb{R}^{4}$ with standard coordinate vectors $e_{1}, e_{2}, e_{3}, e_{4}$. An essential tool will be shear transformations: these are linear maps $\sigma_{i, j}^{a}: \mathbb{R}^{4} \rightarrow \mathbb{R}^{4}$ for $i, j \in\{1,2,3,4\}, i \neq j$, and $a \in \mathbb{R}$ whose matrix is $I_{4}+a \delta_{i, j}$ with respect to the standard basis of $\mathbb{R}^{4}$. Here $I_{4}$ is the $4 \times 4$ unit matrix and $\delta_{i, j}$ is the $4 \times 4$ matrix whose only nonzero entry is a 1 in position $(i, j)$. In particular, $\sigma_{i, j}^{a}$ maps $e_{i}$ to $e_{i}+a e_{j}$, and the standard basis vectors $e_{k}, k \neq i$, to themselves. 

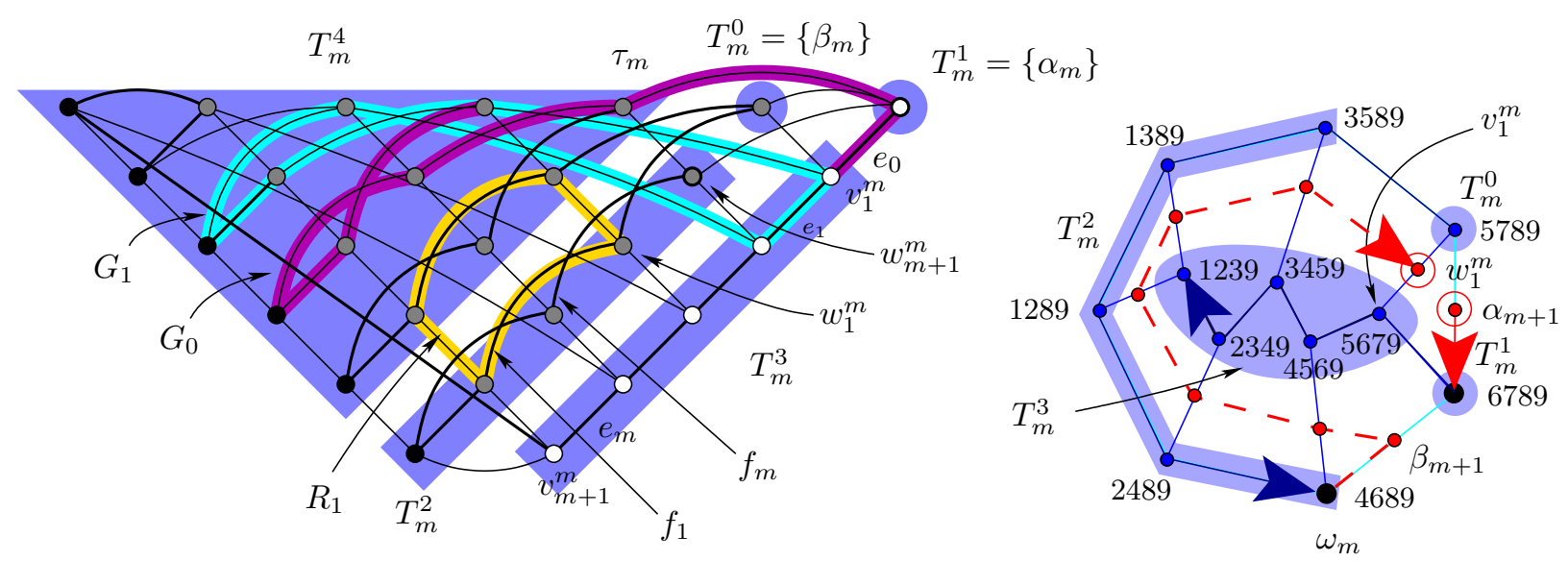

Figure 9: Left: More details about the graph of $Q_{m}$. We have highlighted the graphs of the 2-faces $G_{0}$ and $G_{1}$ that correspond to the edges $e_{0}$ and $e_{1}$ by Proposition $5.2(\mathrm{~b})$, and the 2-face $R_{1}$ that corresponds to the edge $f_{1}$ according to Proposition 5.2 (c). Right: The portion of the new Hamilton path $\widetilde{\pi}_{m+1}$ in the facet $F_{m}^{3}$.

The start of the induction is the pair $\left(Q_{0}, \mathcal{F}_{0}\right)$, where $Q_{0}$ is the 4-simplex whose vertices $v_{1}, v_{2}, v_{3}, v_{4}, v_{5}$ are given by the columns of the matrix

$$
\left(\begin{array}{ccccc}
0 & 0 & 1 & 0 & 0 \\
0 & 1 & 0 & 0 & 0 \\
-3 & -1 & 3 & 2 & 1 \\
-2 & -1 / 2 & 0 & 1 / 4 & 2
\end{array}\right)
$$

and $\mathcal{F}$ is the flag $\mathcal{F}_{0}: F_{0}^{0} \subset F_{0}^{1} \subset \cdots \subset F_{0}^{4}=Q_{0}^{4}$ of faces labeled as in Definition 3.3. In particular, the vertices $v_{i}$ lie in the following tips,

\begin{tabular}{c|c|c|c|c}
$v_{1}$ & $v_{2}$ & $v_{3}$ & $v_{4}$ & $v_{5}$ \\
\hline$T_{0}^{1}$ & $T_{0}^{3}$ & $T_{0}^{4}$ & $T_{0}^{0}$ & $T_{0}^{2}$
\end{tabular},

$F_{0}^{2}=\operatorname{conv}\left\{v_{1}, v_{4}, v_{5}\right\}, F_{0}^{3}=\operatorname{conv}\left\{v_{1}, v_{2}, v_{4}, v_{5}\right\}$, and $\pi_{0}=\left(v_{1}, v_{2}, v_{3}, v_{4}, v_{5}\right)$.

For all $m \geq 0$ the polytopes $Q_{m}$ will maintain the following property:

(M1) The Hamilton path $\pi_{m}$ in the 1-skeleton of $Q_{m}$ is strictly monotone with respect to the objective function $f: \mathbb{R}^{4} \rightarrow \mathbb{R}, \boldsymbol{x} \mapsto x_{4}$.

5.4. Induction step I: Positioning the polytope. In this and the following section, we will position the polytope $Q_{m}$ in such a way that the coordinate subspaces of $\mathbb{R}^{4}$ are compatible with the flag $\mathcal{F}_{m}$. More precisely,

$\triangleright F_{m}^{3}=Q_{m} \cap\left\{\boldsymbol{x} \in \mathbb{R}^{4}: x_{1}=0\right\}$, and $T_{m}^{4} \subset\left\{\boldsymbol{x} \in \mathbb{R}^{4}: x_{1}>0\right\} ;$ and

$\triangleright$ the hyperplane $H_{S}=\left\{\boldsymbol{x} \in \mathbb{R}^{4}: x_{3}=0\right\}$ will separate $T_{\text {even }}^{\leq 4}(m)$ from $T_{\text {odd }}^{\leq 4}(m)$. 
Lemma 5.4. Let $\pi$ be the linear projection $\pi: \mathbb{R}^{4} \rightarrow \mathbb{R}\left\langle e_{3}, e_{4}\right\rangle$, and use the notation of Convention 5.1 and Proposition 5.2(a). Then there exists a non-singular affine transformation $\sigma$ of $\mathbb{R}^{4}$ such that $Q_{m} \equiv \sigma\left(Q_{m}\right)$ satisfies the following additional conditions, while $\pi_{m} \equiv \sigma\left(\pi_{m}\right)$ still satisfies (M1):

(M2) $F_{m}^{2} \subset\left\{\boldsymbol{x} \in \mathbb{R}^{4}: x_{1}=0\right\}$.

(M3) aff $F_{m}^{3}=\left\{\boldsymbol{x} \in \mathbb{R}^{4}: x_{1}=0\right\}$ and $Q_{m} \subset\left\{\boldsymbol{x} \in \mathbb{R}^{4}: x_{1} \geq 0\right\}$.

(M4) $\left(\alpha_{m}\right)_{2}=0, r_{2}<0$ for all $r \in F_{m}^{2} \backslash\left\{\alpha_{m}\right\}$, and $\left(\beta_{m}\right)_{2}<\left(v_{1}^{m}\right)_{2}$.

(M5) The image of $F_{m}^{2}$ under $\pi$ is full-dimensional: $\operatorname{dim}$ aff $\left(\pi\left(F_{m}^{2}\right)\right)=2$.

(M6) The 3-flat $H_{S}=\left\{\boldsymbol{x} \in \mathbb{R}^{4}: x_{3}=0\right\}$ strictly separates $T_{\text {even }}^{\leq 4}(m)$ from $T_{\text {odd }}^{\leq 4}(m)$. Moreover, we may choose the point of $H_{S} \cap F_{m}^{3}$ of lowest 4-coordinate to be $\alpha_{m+1}=$ $\operatorname{conv}\left\{\alpha_{m}, \beta_{m}\right\} \cap H_{S}$, where $\left(\alpha_{m+1}\right)_{4}=\tau_{4}:=\left(\tau_{m}\right)_{4}$.

Proof. Properties (M2) and (M3) are a matter of trivial affine transforms that can be chosen to leave the 4-coordinates invariant, thereby maintaining (M1), and property (M4) can be achieved via a translation and a shear $\sigma_{2,4}^{a}: x_{2} \mapsto x_{2}+a x_{4}$.

For (M5), choose $t \in F_{m}^{2}$ with $t_{4}=q_{4}$ for some $q \in T_{m}^{3}$; such a point exists, since $\alpha_{m} \in F_{m}^{2}$, and $\left(\alpha_{m}\right)_{4}<q_{4}^{\prime}<\max \left\{s_{4}: s \in F_{m}^{2}\right\}$ for all $q^{\prime} \in T_{m}^{3}$ by (M1) and Remark 2.4. Translate $t$ such that $t=\left(0, t_{2}, 0,0\right)$ with $t_{2}<0$, and apply a shear transform $\sigma_{3,2}^{b}: x_{3} \mapsto x_{3}+b x_{2}$ to $\mathbb{R}^{4}$, where $b \in \mathbb{R}$ is chosen such that $\pi\left(\sigma_{3,2}^{b}(q)\right)=\pi\left(\sigma_{3,2}^{b}(t)\right)$. This can be done because $\pi(t)-\pi(q) \in \mathbb{R} \pi\left(e_{3}\right)$. Then (M5) is fulfilled because $\operatorname{dim}$ aff $F_{m}^{3}=3$ : supposing that $\operatorname{dim}$ aff $\left(\pi\left(F_{m}^{2}\right)\right)=1$ would imply via $t \in F_{m}^{2}$ and $q \in F_{m}^{3}$ that $q \in$ aff $F_{m}^{2}$; however, this is absurd by the choice $q \in T_{m}^{3}$. Note that none of the maps we used affects (M2)-(M4).

For (M6), define $\tilde{b}$ to be the point of greatest 3-coordinate of $F_{m}^{2} \cap\left\{\boldsymbol{x} \in \mathbb{R}^{4}: x_{4}=\tau_{4}\right\}$. In particular, $\tilde{b}_{4}>\max _{z \in T^{3}} z_{4}$ by (M1), and $\tilde{b}$ lies either on the edge $\operatorname{conv}\left\{\alpha_{m}, \beta_{m}\right\}$ or on the edge $\operatorname{conv}\left\{\alpha_{m}, \omega_{m}\right\}$ of $F_{m}^{2} \subset Q_{m}$ (cf. Figure 10).

Possibly using the transform $x_{3} \mapsto-x_{3}$, we can achieve $\tilde{b} \in \operatorname{conv}\left\{\alpha_{m}, \beta_{m}\right\}$, and $\tilde{b}=\alpha_{m+1}$ after a translation along the 3 -axis. Now choose a non-horizontal line $\ell$ through $\alpha_{m+1}$ such that $\pi(\ell)$ separates $\pi\left(T_{m}^{1} \cup T_{m}^{3}\right)$ from $\pi\left(F_{m}^{2} \backslash T_{m}^{1}\right)$ (for example, perturb $\ell=\alpha_{m+1}+\mathbb{R} e_{3}$ ), translate $Q_{m}$ again such that $\alpha_{m+1}=0$, and apply a shear $\sigma_{3,4}^{c}: x_{3} \mapsto x_{3}+c x_{4}$ to $\mathbb{R}^{4}$ such that $\ell^{\prime}:=\sigma_{3,4}^{c}(\ell)=\left\{\boldsymbol{x} \in \mathbb{R}^{4}: x_{1}=x_{3}=0\right\} \cap$ aff $F_{m}^{2}$ is vertical, and $x_{3}<0<y_{3}$ for all $x \in T_{m}^{1} \cup T_{m}^{3}$ and $y \in F_{m}^{2} \backslash T_{m}^{1}$ (cf. Figure 10). If the hyperplane $\pi^{-1}\left(\pi\left(\ell^{\prime}\right)\right)$ does not yet separate $T_{m}^{1}$ from $T_{m}^{4}$, apply another shear $\sigma_{3,1}^{d}: x_{3} \mapsto x_{3}+d x_{1}$ with $d>0$ until it does (note that (M3) already holds), and then define $H_{S}:=\pi^{-1}\left(\pi\left(\ell^{\prime}\right)\right)$. This hyperplane then separates the odd and even parts of $\pi_{m}$ by construction, and $\left(\alpha_{m+1}\right)_{4}=\tau_{4}$ also by construction and because the shears $\sigma_{3,4}^{c}$ and $\sigma_{3,1}^{d}$ do not affect 4-coordinates. Neither do they affect conditions (M1)-(M5), so we define $\sigma$ as the composition of all these maps.

Remark 5.5. The conditions (M1)-(M6) are satisfied by the coordinates (5) for $Q_{0}$.

5.5. Induction step II: Finding the cutting plane. In this section, we will find a hyperplane $H_{m+1}$ that gives rise to a polytope $Q_{m+1}^{\prime}=Q_{m} \cap H_{m+1}^{\geq 0}$ of the same combinatorial 


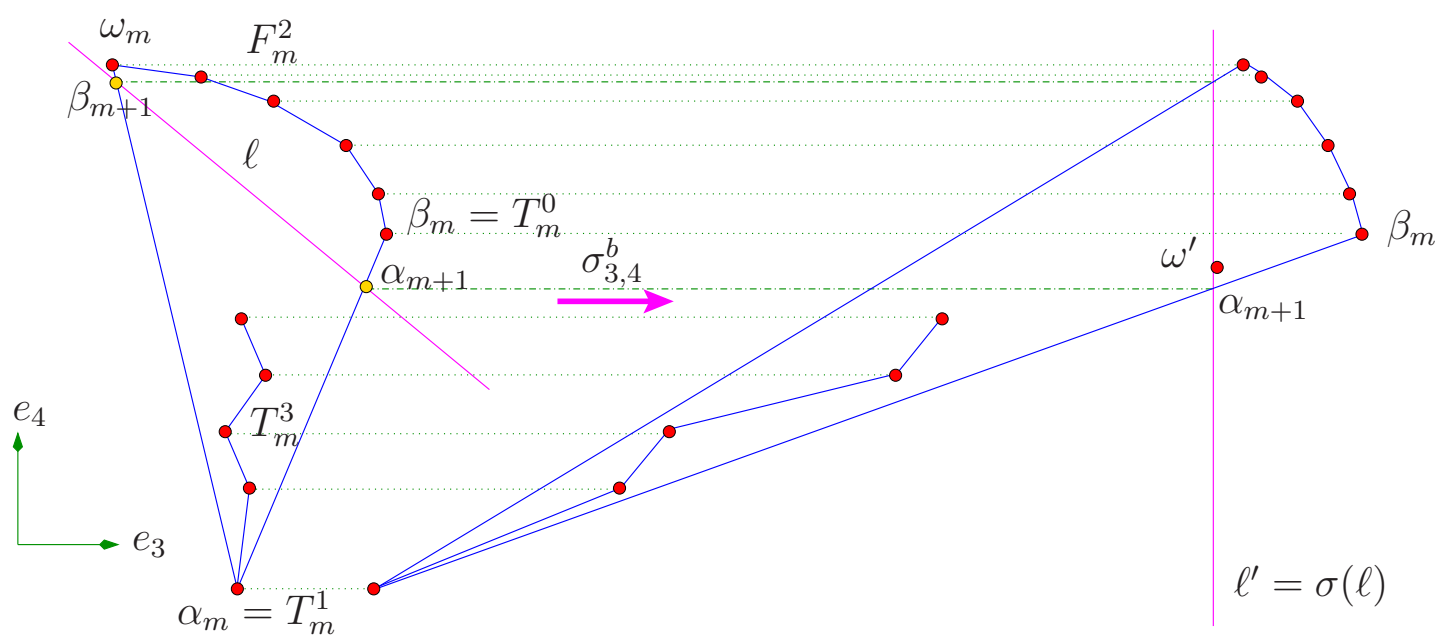

Figure 10: Positioning the polytope, step (M6). The map $\sigma_{3,4}^{b}$ shears the polytope until (the preimage under $\pi$ of) a vertical line $\ell^{\prime}$ separates the odd from the even tips. On the right, the approximate position of $\omega^{\prime}$ is marked; cf. Lemma 5.7.

type as $\widetilde{Q}_{m+1}$. Namely, assume that (M1)-(M6) hold, define $H_{m+1}$ to be the hyperplane $\left\{\boldsymbol{x} \in \mathbb{R}^{4}: \boldsymbol{n}^{T} \boldsymbol{x}=0\right\}$ with $\boldsymbol{n}=(0,-\delta, 1, \varepsilon)^{T}$ for some small $\varepsilon \gg \delta>0$, and assign the label $n+1=m+d+2$ to $H_{m+1}$. Note that $H_{m+1}$ converges to $H_{S}$ as $\varepsilon, \delta \rightarrow 0$.

Remark 5.6. Up to now, we have put the facet $F_{m}^{3}$ into the 3 -plane $\left\{\boldsymbol{x} \in \mathbb{R}^{4}: x_{1}=0\right\}$ and the tip $T^{4}$ into the half-space $\left\{\boldsymbol{x} \in \mathbb{R}^{4}: x_{1}>0\right\}$. This allows us to move "almost all" of the vertices of $\pi_{m}$ (namely, the portion inside $T_{m}^{4}$ ) "out of the way", via a shear $\sigma_{3,1}^{a}$ that only affects 3-coordinates. These "old" vertices will be dealt with in Lemma 5.8 below.

We still need to arrange for the first and last part of $\pi_{m+1}$ to be traversed in the right order. We achieve this by adjusting the position of $H_{m+1}$ via the parameters $\varepsilon$ and $\delta$ in the definition of $\boldsymbol{n}$ (note that we chose $n_{1}=0$, because we are already done with $T_{m}^{4}$ ). If $\delta=0$, then $\pi\left(H_{m+1}\right)$ is a line whose slope is determined by $\varepsilon$. We choose $\varepsilon>0$ to 'push out' the first part $T_{m+1}^{1} \cup T_{m+1}^{3}$ of the new path $\pi_{m+1}$. However, if we left $\delta=0$ we would not correctly sweep the last portion $T_{m+1}^{0} \cup T_{m+1}^{2}$. Items (M8)-(M10) of Lemma 5.7 guarantee a correct sweep in Lemma 5.8 for sufficiently small $0<\delta \ll \varepsilon$.

Lemma 5.7. Assume conditions (M1)-(M6) and $\left(\alpha_{m+1}\right)_{3}=\left(\alpha_{m+1}\right)_{4}=0$, and fix vertices $q \in T_{\text {odd }}^{\leq 4}(m)$ and $s \in T_{\text {even }}^{\leq 4}(m)$. Let $q^{\prime}=\operatorname{conv}\{q, s\} \cap H_{m+1}$ be the intersection with $H_{m+1}$ of the line through $q$ and $s$ (which is not necessarily an edge of $Q_{m}$ ). Then, if $a>0$ is sufficiently large and $0<\delta \ll \varepsilon$ are sufficiently small, the image $\sigma_{3,1}^{a}\left(Q_{m}\right)$ of $Q_{m}$ under the shear $\sigma_{3,1}^{a}$ satisfies the following conditions (M7)-(M10); cf. also Figure 12 below.

(M7) $q_{3}^{\prime}>0$ for $0<\delta \ll \varepsilon$, and $q_{3}^{\prime} \searrow 0$ as $\delta, \varepsilon \searrow 0$. In other words, all points in $\sigma_{3,1}^{a}\left(Q_{m}\right) \cap H_{m+1}$ can be chosen to have positive 3-coordinate, but to lie arbitrarily close to $H_{S}$. 
(M8) Set $u:=\left(v_{1}^{m+1}\right)^{\prime}=\operatorname{conv}\left\{\alpha_{m}, \tau_{m}\right\} \cap H_{m+1}$ and suppose that $q^{\prime} \neq u$. Then the image $\pi\left(\right.$ aff $\left.\left\{u, q^{\prime}\right\}\right) \subset \mathbb{R}\left\langle e_{3}, e_{4}\right\rangle$ of the line through $u$ and $q^{\prime}$ under $\pi$ comes arbitrarily close to being vertical as $a \rightarrow \infty$ and $\varepsilon, \delta \rightarrow 0$.

(M9) Set $\alpha^{\prime}:=\alpha_{m+1}^{\prime}=\operatorname{conv}\left\{\alpha_{m}, \beta_{m}\right\} \cap H_{m+1}$. If $q, \bar{q} \in T_{m}^{3}$ and $q_{4}<\bar{q}_{4}$, so that $q^{\prime}, \bar{q}^{\prime} \in T_{m+1}^{3}$ and $q_{4}^{\prime}<\bar{q}_{4}^{\prime}$, then the slope $\sigma_{\alpha^{\prime} \bar{q}^{\prime}}$ of the line $\pi\left(\right.$ aff $\left.\left\{\alpha^{\prime}, \bar{q}^{\prime}\right\}\right)$ is greater than the slope $\sigma_{\alpha^{\prime} q^{\prime}}$ of the line $\pi\left(\operatorname{aff}\left\{\alpha^{\prime}, q^{\prime}\right\}\right)$ (and both are negative).

(M10) Set $\omega^{\prime}:=\omega_{m+1}^{\prime}=\operatorname{conv}\left\{\beta_{m}, v_{1}^{m}\right\} \cap H_{m+1}$. Then the slope $\sigma_{\omega^{\prime} \alpha^{\prime}}$ of $\pi\left(\right.$ aff $\left.\left\{\omega^{\prime}, \alpha^{\prime}\right\}\right)$ is less than the slope $\sigma_{\omega^{\prime} u}$ of $\pi\left(\operatorname{aff}\left\{\omega^{\prime}, u\right\}\right)$.

Proof. We abbreviate $\sigma=\sigma_{3,1}^{a}$. For (M7), we have $\operatorname{conv}\{q, s\} \cap H_{m+1} \neq \emptyset$ since $q$ and $s$ are separated by $H_{m+1}$ for small enough $\delta, \varepsilon$. We calculate the intersection point $q^{\prime}=$ $\operatorname{conv}\{q, s\} \cap H_{m+1}$ by solving $\boldsymbol{n}^{T} \boldsymbol{q}+\mu \boldsymbol{n}^{T}(\boldsymbol{s}-\boldsymbol{q})=0$ for $\mu$, obtaining

$$
\boldsymbol{q}^{\prime}=\boldsymbol{q}+\frac{\boldsymbol{n}^{T} \boldsymbol{q}}{\boldsymbol{n}^{T}(\boldsymbol{q}-\boldsymbol{s})}(\boldsymbol{s}-\boldsymbol{q})
$$

By (M2), the map $\sigma$ leaves the points $\alpha^{\prime}, q$, and $\omega^{\prime}$ invariant, and maps $\boldsymbol{s}$ to $\sigma(\boldsymbol{s})=\boldsymbol{s}+a s_{1} \boldsymbol{e}_{3}$; as a consequence, $\boldsymbol{n}^{T} \sigma(\boldsymbol{s})=\boldsymbol{n}^{T} \boldsymbol{s}+a s_{1}$. Using $\boldsymbol{n}^{T} \boldsymbol{q}=-\delta q_{2}+q_{3}+\varepsilon q_{4}$, we obtain

$$
\begin{aligned}
\sigma\left(\boldsymbol{q}^{\prime}\right)=\boldsymbol{q}+\frac{\boldsymbol{n}^{T} \boldsymbol{q}}{\boldsymbol{n}^{T}(\boldsymbol{q}-\boldsymbol{s})-a s_{1}}\left(\boldsymbol{s}-\boldsymbol{q}+a s_{1} \boldsymbol{e}_{3}\right) \\
\underset{a \rightarrow \infty}{\longrightarrow} \boldsymbol{q}+\left(0,0,-\boldsymbol{n}^{T} \boldsymbol{q}, 0\right)^{T}=\left(0, q_{2}, \delta q_{2}-\varepsilon q_{4}, q_{4}\right)^{T} .
\end{aligned}
$$

Because $q_{4}<\left(\alpha_{m+1}\right)_{4}=0$, we can choose $0<\delta \ll \varepsilon$ so small that $\sigma\left(\boldsymbol{q}^{\prime}\right)_{3}>0$ (note that $q_{2} \leq 0$ by $\left.(\mathrm{M} 4)\right)$. In particular, we obtain $\sigma\left(\boldsymbol{q}^{\prime}\right)_{3} \searrow 0$ as $\varepsilon, \delta \searrow 0$.

Statement (M8) follows from (6) and the fact that

$$
\lim _{a \rightarrow \infty} \frac{\sigma\left(\boldsymbol{q}^{\prime}\right)_{4}-\sigma(\boldsymbol{u})_{4}}{\sigma\left(\boldsymbol{q}^{\prime}\right)_{3}-\sigma(\boldsymbol{u})_{3}}=\frac{q_{4}-u_{4}}{\delta\left(q_{2}-u_{2}\right)-\varepsilon\left(q_{4}-u_{4}\right)} .
$$

For (M9), note that since $\alpha^{\prime}$ is invariant under $\sigma$,

$$
\sigma_{\alpha^{\prime} q^{\prime}}=\frac{\sigma\left(\boldsymbol{q}^{\prime}\right)_{4}-\alpha_{4}^{\prime}}{\sigma\left(\boldsymbol{q}^{\prime}\right)_{3}-\alpha_{3}^{\prime}} \quad \underset{a \rightarrow \infty}{\longrightarrow} \frac{q_{4}-\alpha_{4}^{\prime}}{\delta q_{2}-\alpha_{3}^{\prime}-\varepsilon q_{4}},
$$

and similarly for $\bar{q}$; the statement now follows from $q_{4}<\bar{q}_{4}$ and $0<\delta \ll \varepsilon$.

To prove (M10), set $\alpha:=\alpha_{m}, \beta:=\beta_{m}, v:=v_{1}^{m}$ and $\tau:=\tau_{m}$. Then $u=\operatorname{conv}\{\alpha, \tau\} \cap$ $H_{m+1}, \alpha^{\prime}=\operatorname{conv}\{\alpha, \beta\} \cap H_{m+1}$, and $\omega^{\prime}=\operatorname{conv}\{v, \beta\} \cap H_{m+1}$. We need to verify that

$$
\sigma_{\omega^{\prime} \alpha^{\prime}}:=\frac{\alpha_{4}^{\prime}-\omega_{4}^{\prime}}{\alpha_{3}^{\prime}-\omega_{3}^{\prime}}<\frac{u_{4}-\omega_{4}^{\prime}}{u_{3}-\omega_{3}^{\prime}}=: \sigma_{\omega^{\prime} u} .
$$


From equation (6) and condition (M4), we deduce that $\lim _{a \rightarrow \infty} u=\left(0,0,-\varepsilon \alpha_{4}, \alpha_{4}\right)^{T}$. For $\alpha^{\prime}$ and $\omega^{\prime}$ we get the following expressions:

$$
\begin{gathered}
\boldsymbol{\alpha}^{\prime}=\boldsymbol{\alpha}+\frac{\boldsymbol{n}^{T} \boldsymbol{\alpha}}{\boldsymbol{n}^{T}(\boldsymbol{\alpha}-\boldsymbol{\beta})}(\boldsymbol{\beta}-\boldsymbol{\alpha})=\left(\begin{array}{c}
0 \\
0 \\
\alpha_{3} \\
\alpha_{4}
\end{array}\right)+\frac{\alpha_{3}+\varepsilon \alpha_{4}}{\delta \beta_{2}+\alpha_{3}-\beta_{3}+\varepsilon\left(\alpha_{4}-\beta_{4}\right)}\left(\begin{array}{c}
0 \\
\beta_{2} \\
\beta_{3}-\alpha_{3} \\
\beta_{4}-\alpha_{4}
\end{array}\right), \\
\boldsymbol{\omega}^{\prime}=\boldsymbol{v}+\frac{\boldsymbol{n}^{T} \boldsymbol{v}}{\boldsymbol{n}^{T}(\boldsymbol{v}-\boldsymbol{\beta})}(\boldsymbol{\beta}-\boldsymbol{v})=\left(\begin{array}{c}
0 \\
v_{2} \\
v_{3} \\
v_{4}
\end{array}\right)+\frac{-\delta v_{2}+v_{3}+\varepsilon v_{4}}{-\delta\left(v_{2}-\beta_{2}\right)+v_{3}-\beta_{3}+\varepsilon\left(v_{4}-\beta_{4}\right)}\left(\begin{array}{c}
0 \\
\beta_{2}-v_{2} \\
\beta_{3}-v_{3} \\
\beta_{4}-v_{4}
\end{array}\right) .
\end{gathered}
$$

For convenience, we will verify that $1 / \sigma_{\omega^{\prime} \alpha^{\prime}}>1 / \sigma_{\omega^{\prime} u}$. Indeed, expanding these expressions in terms of $\delta, \varepsilon$, we obtain

$$
\begin{aligned}
\frac{1}{\sigma_{\omega^{\prime} \alpha^{\prime}}} & =\frac{\beta_{3} v_{2}-\beta_{2} v_{3}+\overbrace{\alpha_{3}\left(\beta_{2}-v_{2}\right)}^{t_{1}}}{v_{3}\left(\alpha_{4}-\beta_{4}\right)+\beta_{3}\left(v_{4}-\alpha_{4}\right)+\underbrace{\alpha_{3}\left(\beta_{4}-v_{4}\right)}_{t_{2}}} \delta-\varepsilon+p_{1}(\delta, \varepsilon), \\
\frac{1}{\sigma_{\omega^{\prime} u}} & =\frac{\beta_{3} v_{2}-\beta_{2} v_{3}}{v_{3}\left(\alpha_{4}-\beta_{4}\right)+\beta_{3}\left(v_{4}-\alpha_{4}\right)} \delta-\varepsilon+p_{2}(\delta, \varepsilon),
\end{aligned}
$$

where $p_{1}$ and $p_{2}$ are power series in $\delta, \varepsilon$ with min-degree at least 2 . Notice that up to terms of degree at least 2 in $\delta, \varepsilon$, the two formulas are equal except for the expressions $t_{1}$ resp. $t_{2}$ in the numerator resp. denominator of $1 / \sigma_{\omega^{\prime} \alpha^{\prime}}$. Therefore, we can write the difference between the inverses of the slopes as

$$
\frac{1}{\sigma_{\omega^{\prime} \alpha^{\prime}}}-\frac{1}{\sigma_{\omega^{\prime} u}}=\left(\frac{A+t_{1}}{B+t_{2}}-\frac{A}{B}\right) \delta+p_{3}(\delta, \varepsilon) .
$$

Since $\alpha_{3}<\left(\alpha_{m+1}\right)_{3}<0$ by assumption and $\beta_{2}<v_{2}$ by (M4), we obtain $t_{1}>0$; and the inductive assumption (M1) implies that $\beta_{4}>v_{4}$ and therefore $t_{2}<0$. The claim follows.

5.6. Induction step III: The projective transformation. Finally, we construct a 1parameter family $\mathcal{H}=\left\{H_{t}: t \in \mathbb{P}^{1}(\mathbb{R})\right\}$ of hyperplanes that contains a 2-plane $R$ as their common "axis", as in Section 5.1. Let $O=\pi\left(b+\varepsilon_{1}(\omega-\alpha)-\varepsilon_{3} e_{3}\right)$ for some small $\varepsilon_{1}, \varepsilon_{3}>0$, so that $O$ lies outside but very close to the edge $\operatorname{conv}\{\alpha, \omega\}$ of $\pi\left(F_{m+1}^{2}\right)$, and define the 2-plane $R \subset \mathbb{R}^{4}$ to be $R=\pi^{-1}(O)$.

Lemma 5.8. Let $\mathcal{H}$ be the pencil of hyperplanes in $\mathbb{R}^{4}$ sharing the 2-plane $R$, and such that $\pi\left(H_{\infty}\right)$ is the line through $O$ parallel to $\operatorname{conv}\{\alpha, \omega\}$, and the slope of $\pi\left(H_{r}\right)$ is smaller than the slope of $\pi\left(H_{s}\right)$ exactly if $r<s$. Then $\mathcal{H}$ fulfills (S2), i.e., it sorts the vertices of $Q_{m+1}$ in the order given by $\pi_{m+1}$. 
Proof. We examine the pieces of $\pi_{m+1}$ in order; cf. Figure 12.

$\triangleright T_{m+1}^{1}=\{\alpha\}$ is the start of $\pi_{m+1}$ : This follows for small enough $\varepsilon_{3}$ by (M10).

$\triangleright T_{m+1}^{3}$ is traversed next, in the right order, and before $T_{m+1}^{4}$ : The first two statements follow from (M7), (M8) and (M9), and the last one because $z_{3} \rightarrow \infty$ as $a \rightarrow \infty$ for any $z \in T_{m}^{4}$, while the 3 -coordinates of $T_{m+1}^{3}$ remain bounded by (M7).

$\triangleright$ The correct order in $T_{m}^{4} \subset T_{m+1}^{4}$. By Proposition 5.2(b), each of the edges $e_{i}=$ $\operatorname{conv}\left\{v_{i}^{m}, v_{i+1}^{m}\right\}, 0 \leq i \leq m$, of $T_{m}^{1} \cup T_{m}^{3}$ is incident to an $(m+1)$-gonal 2-face $G_{i}$ (see Figure 9), and the edges $E_{i}$ of $G_{i}$ not incident to $e_{i}$ form a monotone subpath of $\pi_{m+1}$. This implies that for each $e_{i} \in T_{m}^{3}$, the slopes of the projection of each $E_{i}$ to $\mathbb{R}\left\langle e_{3}, e_{4}\right\rangle$ are strictly positive (and, by convexity, monotonically decreasing; see Figure 11).

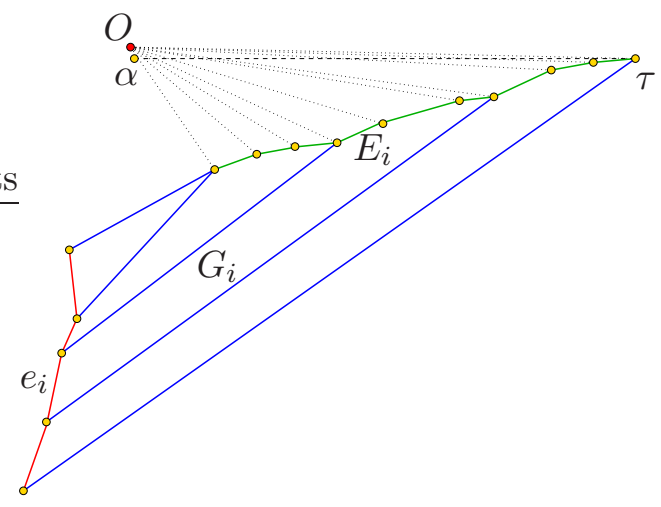

Figure 11: Convexity of the $(m+1)$-gonal faces enforces the correct order in $T_{m}^{4} \subset T_{m+1}^{4}$.

Therefore, $\pi\left(\bigcup_{i=0}^{m} E_{i}\right)$ is a strictly increasing chain of edges, and this remains true after applying the linear map $\sigma=\sigma_{3,1}^{a}$ by invariance of the $e_{i}$ 's and all 4-coordinates under $\sigma$, and the convexity of the projections of 2-faces. The correct order up to $\tau$ in $T_{m}^{4} \subset T_{m+1}^{4}$ follows from condition (M6): $\alpha_{4} \geq s_{4}$ for all $s \in \bigcup_{i=0}^{m} \operatorname{vert} G_{i} \backslash \operatorname{vert} e_{i}$. Similarly, the 4-gonal 2-faces incident to $T_{m}^{2}$ of Proposition 5.2(c) enforce the right order between $\tau$ and $T_{m}^{0}$.

$\triangleright T_{m+1}^{2}$ is traversed after $T_{m+1}^{4}$ : Since $\beta$, the first vertex of $\pi_{m+1}$ to come after $T_{m+1}^{4}$, lies on $\operatorname{conv}\left\{\alpha_{m}, \omega_{m}\right\}$, this can be achieved by choosing $\varepsilon$ and $\varepsilon_{1}$ suitably small.

$\triangleright$ Correct order in $T_{m+1}^{2}$ and $T_{m+1}^{0}$. This follows because the convex polygon $\pi\left(F_{m+1}^{2}\right)$ is star-shaped with respect to any point on its boundary, and the choice of $O$ close to an edge of $\pi\left(F_{m+1}^{2}\right)$.

This concludes the proof of Lemma 5.8.

Finally, we apply the projective transform $\psi: \mathbb{R}^{4} \rightarrow \mathbb{R}^{4}, \boldsymbol{x} \mapsto \boldsymbol{x} /\left(\boldsymbol{a} \boldsymbol{x}-a_{0}\right)$ that sends the 3-plane $H_{\infty}=\left\{\boldsymbol{x} \in \mathbb{R}^{4}: \boldsymbol{a x}=a_{0}\right\}$ to infinity, and set $Q_{m+1}:=\psi\left(Q_{m+1}^{\prime}\right)$. Lemma 5.8 then implies the inductive condition (M1), namely that $Q_{m+1}$ admits an monotone Hamilton path $\pi_{m+1}$. The proof of Theorem 2.5, and so of the Main Theorem, is concluded. 


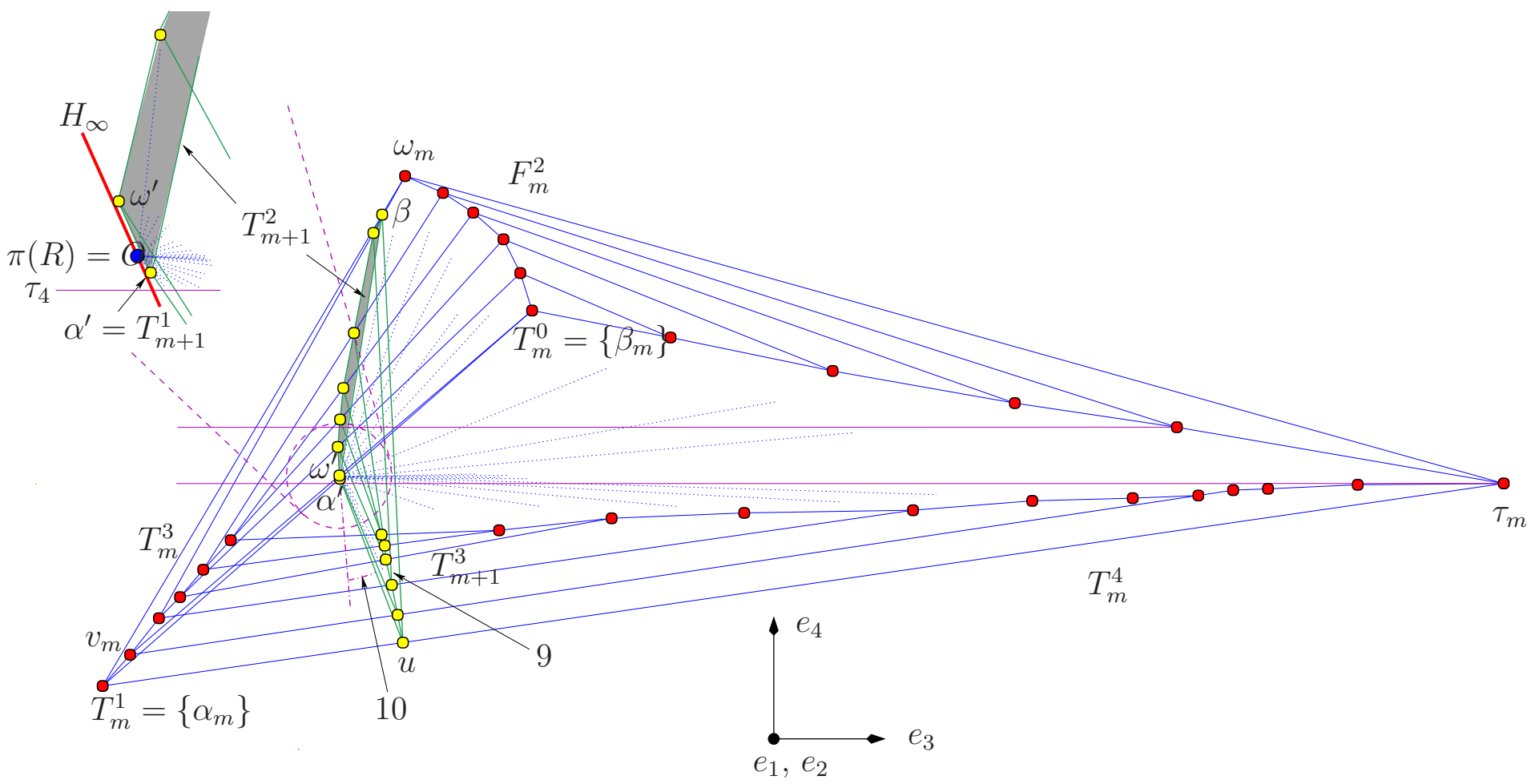

Figure 12: The inductive step: We show the projection of the polytope $Q_{4}$ to the $\langle 3,4\rangle$-plane, and the vertices obtained by intersecting $Q_{4}$ with $H_{5}$. The arrows next to the labels 9 and 10 point to the lines about whose slope the corresponding condition in Lemma 5.7 makes an assertion. The line through $O$ is the projection of the 3-plane $H_{\infty}$. A sweep around $O$ encounters all vertices of $Q_{m} \cap H_{m+1}$ in the correct order $\pi_{m}$ prescribed by $\widetilde{\pi}_{m+1}$. 


\section{Acknowledgements}

It is a pleasure to thank Günter M. Ziegler for suggesting this problem, and Volker Kaibel for his careful reading of an earlier version of the paper.

\section{REFERENCES}

[1] David W. Barnette, A family of neighborly polytopes, Isr. J. Math., 39 (1981), 127-140.

[2] Fred Holt and Victor KleE, A proof of the strict monotone 4-step conjecture, Contemp. Math., 223 (1999), 201-216.

[3] Michael Joswig, Volker Kaibel, and Friederike Körner, On the k-systems of a simple polytope, Isr. J. Math., 129 (2002), 109-117.

[4] Victor KleE, Heights of convex polytopes, J. Math. Anal. Appl., 11 (1965), 176-190.

[5] Peter McMullen, The numbers of faces of simplicial polytopes, Isr. J. Math., 9 (1971), 559-570.

[6] Theodore S. Motzkin, Comonotone curves and polyhedra, Abstract, Bulletin Amer. Math. Soc., 63 (1957), 35 .

[7] Julian Pfeifle and Günter M. Ziegler, On the monotone upper bound problem, Experimental Math., (2004), to appear.

[8] Ido Shemer, Neighborly polytopes, Isr. J. Math., 43 (1982), 291-314.

[9] Günter M. Ziegler, Lectures on Polytopes, Graduate Texts in Mathematics 152, Springer, New York, 1995. Revised edition 1998.

Institut de Matemàtica, Universitat de Barcelona, Gran Via de les Corts Catalanes 585, E-08007 BARCELONA, SPAIN

E-mail address: julian@imub.ub.es 